\title{
The spatial epidemiology of tuberculosis in Linyi City, China, 2005-2010
}

Tao Wang ${ }^{1,2}$, Fuzhong Xue ${ }^{1}$, Yongjin Chen ${ }^{3}$, Yunbo $\mathrm{Ma}^{3}$ and Yanxun Liu' ${ }^{1 *}$

\begin{abstract}
Background: Tuberculosis (TB) remains a major public health burden in many developing countries. China alone accounted for an estimated 12\% of all incident TB cases worldwide in 2010. Several studies showed that the spatial distribution of TB was nonrandom and clustered. Thus, a spatial analysis was conducted with the aim to explore the spatial epidemiology of TB in Linyi City, which can provide guidance for formulating regional prevention and control strategies.

Methods: The study was based on the reported cases of TB, between 2005 and 2010. 35,308 TB cases were geo-coded at the town level $(n=180)$. The spatial empirical Bayes smoothing, spatial autocorrelation and space-time scan statistic were used in this analysis.

Results: Spatial distribution of TB in Linyi City from 2005 to 2010 was mapped at town level in the aspects of crude incidence, excess hazard and spatial smoothed incidence. The spatial distribution of TB was nonrandom and clustered with the significant Moran's / for each year. Local $G_{i}^{*}$ detected five significant spatial clusters for high incidence of TB. The space-time analysis identified one most likely cluster and nine secondary clusters for high incidence of TB.

Conclusions: There is evidence for the existence of statistically significant TB clusters in Linyi City, China. The result of this study may assist health departments to develop a better preventive strategy and increase the public health intervention's effectiveness.
\end{abstract}

\section{Background}

Tuberculosis (TB) is an infectious disease caused by the bacillus Mycobacterium tuberculosis, which is still a leading cause of death in low-income and middleincome countries, and remains a major public health burden in many developing countries [1]. China (0.9 million-1.2 million) alone accounted for an estimated $12 \%$ of all incident TB cases worldwide in 2010 [2]. Linyi City is one of the high-incidence areas in China, and there is an estimated 6,000 reported cases every year from 2005-2010. Thus, preventive strategies are urgently needed in the development of TB.

In recent years, geographical information systems (GIS) and spatial analysis were frequently used to describe the pattern of TB. In India, purely spatial and retrospective space-time analysis were used to find

\footnotetext{
* Correspondence: liu_yx@sdu.edu.cn

${ }^{1}$ Department of Epidemiology and Health Statistics, Shandong University, Jinan 250012, People's Republic of China

Full list of author information is available at the end of the article
}

significant hotspots of TB in three areas of the Almora district [3]. In Japan, space-time scan statistics identified TB clusters in Fukuoka [4]. In Madagascar, spatial clustering of TB was associated with socio-economic and patient care factors in Antananarivo City [5]. With respect to spatiotemporal clustering, three high incidence spacetime clusters were identified in Portugal between 2000 and 2004 [6]. In South African, GIS and spatial analysis were used to study TB transmission patterns in a highincidence area [7]. In an Urban West African, spatial scan statistic was used to assess purely spatial and space-time clusters of TB in Greater Banjul [8]. Another study in Brazil investigated spatial patterns of the incidence of TB and its relationship with socio-economic status [9]. In Beijing, GIS and spatial analysis were used to determine the role of migration in the transmission of TB [10]. To our best knowledge, there were few studies to explore the spatial epidemiology of TB in Linyi City, China. A better understanding of the spatial epidemiology of TB may help health departments to provide 
guidance for formulating regional prevention and control strategies $[9,10]$.

The spatial analyses, such as spatial smoothing, spatial autocorrelation and cluster analysis are commonly used to characterize spatial epidemiology of diseases. Spatial smoothing was used to reduce random variation associated with small populations [9,11-14]. Spatial autocorrelation analysis was performed to detect significantly difference from a random spatial distribution [10,15-17]. Spatial cluster analysis was conducted to identify whether cases of disease were geographically clustered $[4,8,14,18-25]$.

Therefore, we conducted GIS-based spatial analyses involving spatial smoothing, spatial autocorrelation analysis and space-time scan statistic to characterize geographic distribution pattern of TB in Linyi City of China during 2005-2010.

\section{Methods}

\section{Data collection and management}

Relevant data for Linyi City (Figure 1), which is in the south of Shandong Province, was used in the study. Records on TB cases between 2005 and 2010 were obtained from the Linyi Institute for Tuberculosis Control, and we were permitted to use the data. To conduct a GIS-based analysis on the spatial distribution of TB, the town-level polygon map at 1:100,000 scale was obtained, on which the town-level point layer containing information regarding latitudes and longitudes of central points of each county was created. Demographic information based on Linyi Statistical Yearbook was integrated in terms of the administrative code. All TB cases were geo-coded and matched to the town-level layers of polygon and point by administrative code using the software ArcGIS9.3 (ESRI Inc., Redlands, CA, USA).

\section{GIS mapping and smoothing}

To alleviate variations of incidence in small populations and areas, annualized average incidences of $\mathrm{TB}$ per 100,000 at each town over the 6 year-period were calculated, and spatial empirical Bayes smoothing was implemented in SpaceStat software [26].

To assess the risk of TB in each town, an excess hazard map was produced. The excess hazard represents the ratio of the observed incidence at each town over the average incidence of all areas, the later was calculated by the number of cases over the total number of people at risk instead of the annualized incidence of a town [27].

\section{Spatial autocorrelation analysis}

Spatial autocorrelation analyses were performed in SpaceStat. Global Moran's I statistics was used to discern spatial autocorrelation and detect the spatial distribution pattern of TB in Linyi City, China. Local $G_{i}^{*}$ was used to examine the local level of spatial autocorrelation and determine locations of clusters or hotspots. A calculated value of $G_{i}^{*} \geq 1.96$ indicates that the town and its neighboring towns have a TB incidence rate statistically significantly higher than other towns. The number of permutation test was set to 999 and the significance level was set as 0.05 .

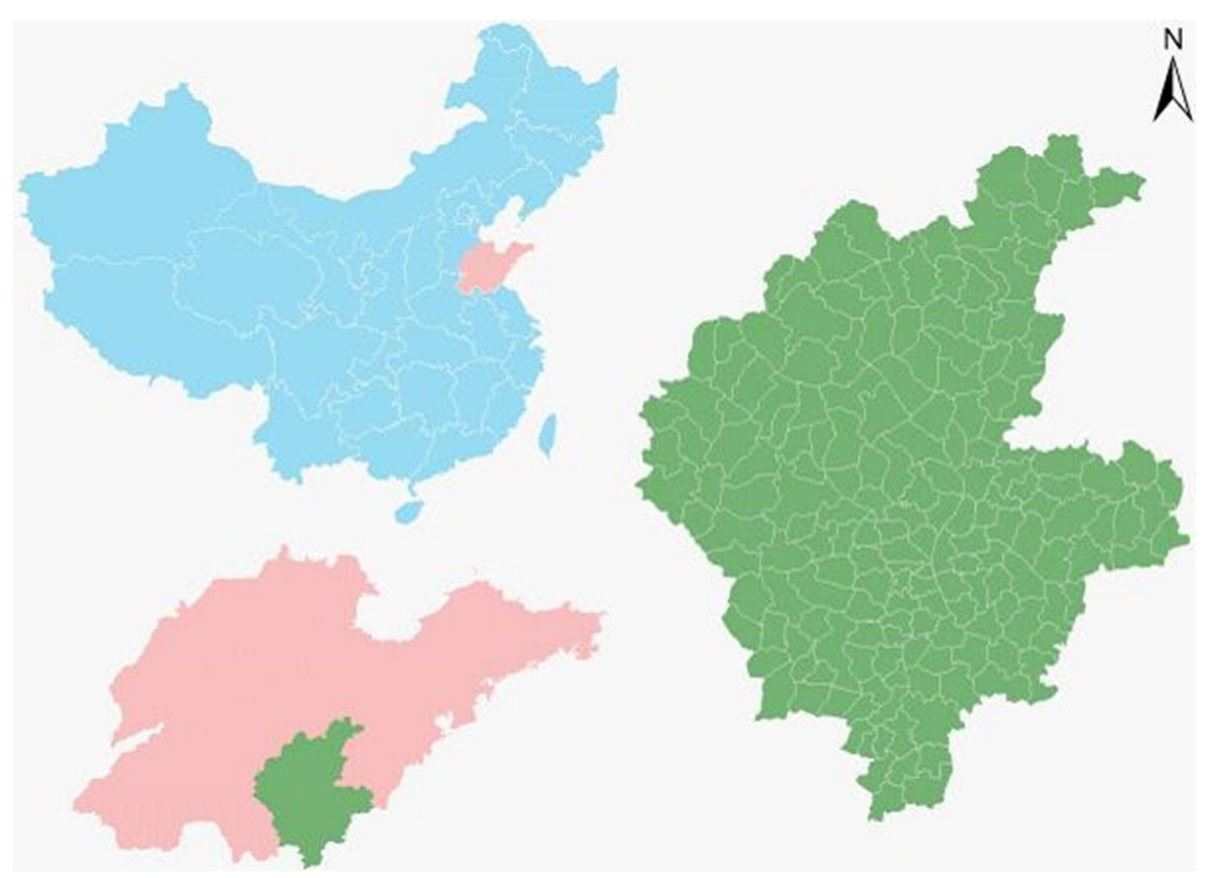

Figure 1 Location of the study area. 


\section{Space-time scan statistic}

The space-time scan statistic was performed using SaTS$\mathrm{can}^{\mathrm{TM}}$ v9.1.1 software [28]. The method is defined by a cylindrical window with a circular geographic base and with height corresponding to time [29]. The null hypothesis assumed that the relative risk (RR) of TB was the same within the window compared to outside.

For this analysis, a Poisson based model was used, where the number of events in an area is Poisson distributed according to a known underlying population at risk [30]. The geographic size of the window was limited to half the expected number of cases and that the time size was limited to half the total time period [29]. The test of significance of the identified clusters was based on comparing the likelihood ratio test statistics against a null distribution obtained from Monte Carlo Simulation [19]. The number of permutation was set to 999 and the significance level was set as 0.05 .

\section{Results}

Descriptive analysis of TB in Linyi City

There were 35,329 TB cases reported in Linyi City, from 2005 to 2010. Of these, 35,308 (99.94\%) had complete information including mapping of their place of residence. Annualized average incidence at the town-level ranged from 27.21 to 100.58 per 100,000 (Figure 2).

The excess hazard map showed distribution of the excess risk, which was defined as a ratio of the observed number over the expected number of cases. Towns in blue color had lower incidences than expected, as indicated by excess risk values less than 1. In contrast, towns in red color had higher incidences than expected or excess risk values greater than 1 (Figure 3).

Spatial empirical Bayes smoothed map for annualized average incidence was created by correcting the variance in the variability of incidence (Figure 4).

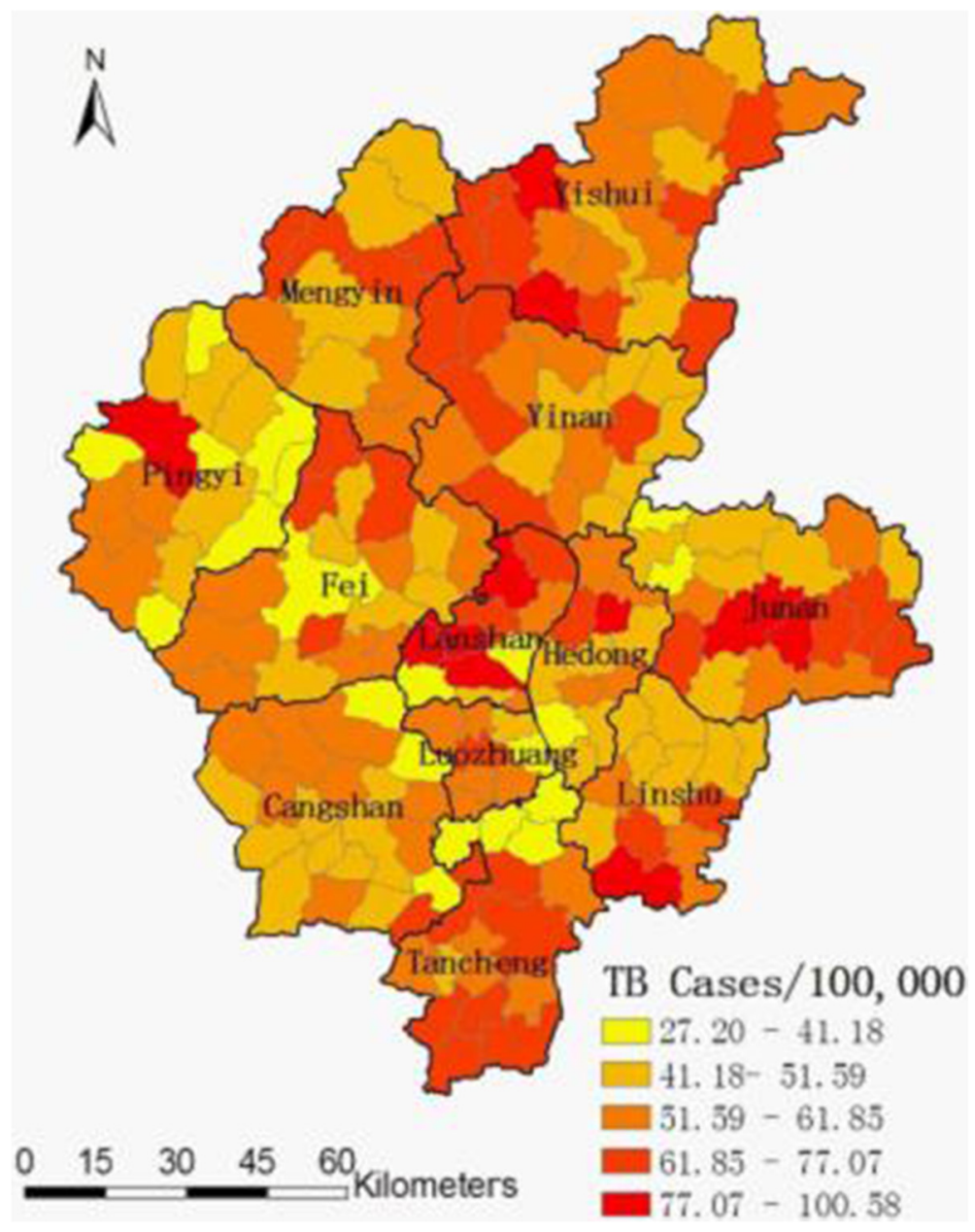

Figure 2 Annualized average incidence of TB in Linyi City during 2005-2010. 


\section{Spatial autocorrelation analysis of TB in Linyi City}

The global spatial autocorrelation analyses for annualized incidence of TB in Linyi City from 2005 to 2010 showed that the Moran's $I$ was significant for each year (Table 1), implying that distribution of TB was spatially autocorrelated in Linyi City, China.

Five significant spatial clusters (hotspots) of TB were identified using the Local $G_{i}^{*}$ for spatial autocorrelation (Table 2 and Figure 5). The hotspots persisted in Pingyi Sub-district, Gaozhuang Town, Xiawei Town, Mamuchi Town, Pingshang Town, Zhuanggang Town, Fangqian Town, Xiangdi Town, Zhubian Town, Shizilu Subdistrict, Gaofengtou Town, Guichang Town, Lanshan Sub-district, Baishabu Town, Yitang Town, and Zaogoutou Town from 2005 to 2010.

\section{Space-time analysis of TB in Linyi City}

Space-time cluster analysis of TB in 2005-2010 in Linyi City showed that TB was not distributed randomly in space-time. The most likely statistically significant cluster for high incidence of TB was found to exist at Pingyi Subdistrict, for the year 2008-2010 ( $R R=2.22, p<0.001)$, with 520 observed cases and 235.93 expected cases. Nine statistically significant secondary clusters were also detected for high incidence of TB. The results are listed in Table 3, and depicted on the map in Figure 6.

\section{Discussion}

In our study, exploratory spatial data analysis and spatial cluster analysis of TB were conducted at town level in Linyi City, China. We mapped TB from different aspects such as crude incidence, excess risk, and spatial empirical Bayes smoothed incidence, investigated the spatial pattern and highlighted geographic areas with significant high incidence of TB in Linyi. The study showed that the spatial distribution of TB in Linyi City was nonrandom and clustered with the significant Moran's $I$ for each year. Local $G_{i}^{*}$ detected five significant spatial clusters for high incidence of TB when only space distribution was considered. However, one most likely cluster and nine secondary clusters for high incidence of $\mathrm{TB}$ were identified when both space and time were considered in the space-time analysis. When compared the

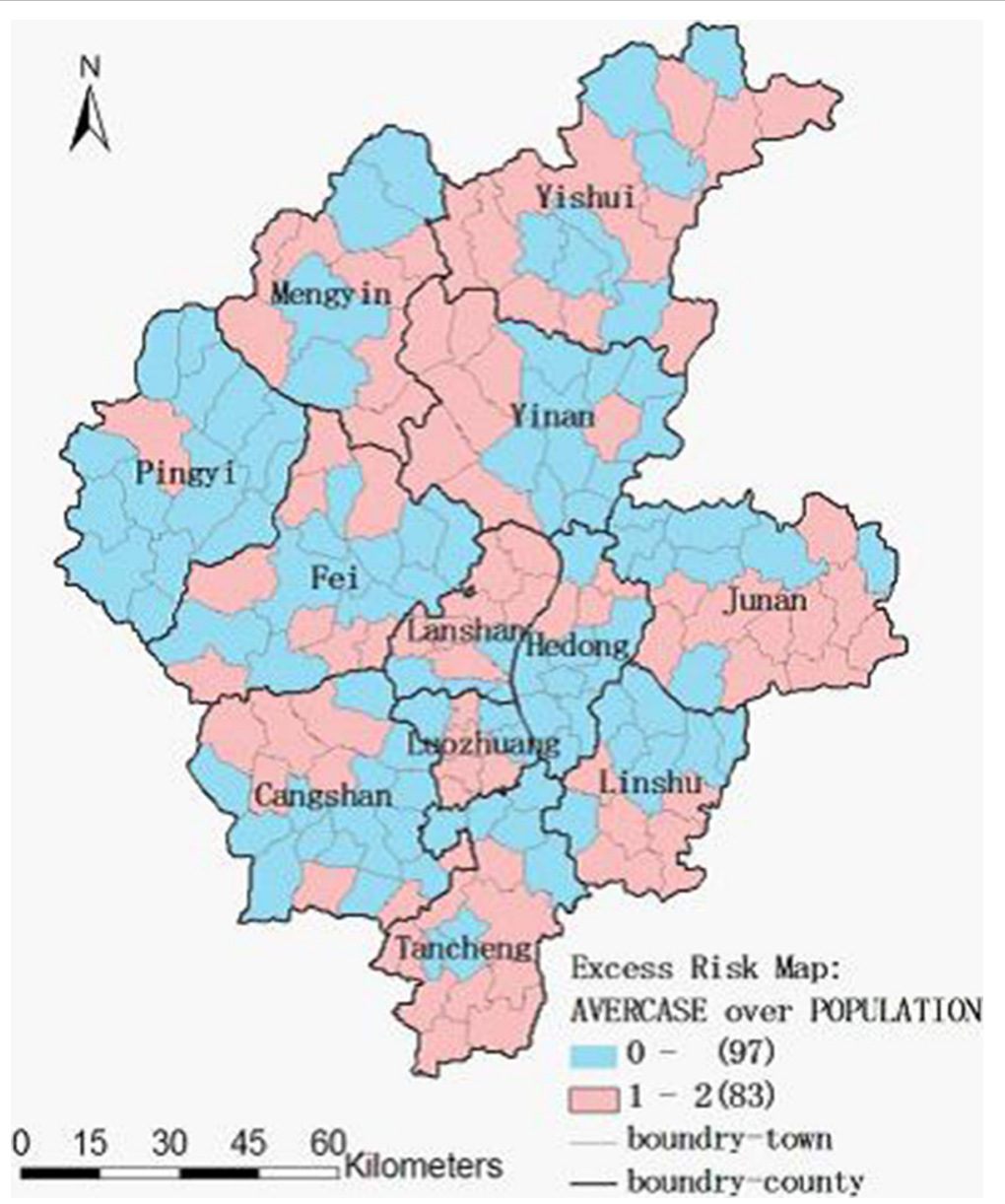

Figure 3 Excess hazard map of TB in Linyi City, China from 2005 to 2010. 


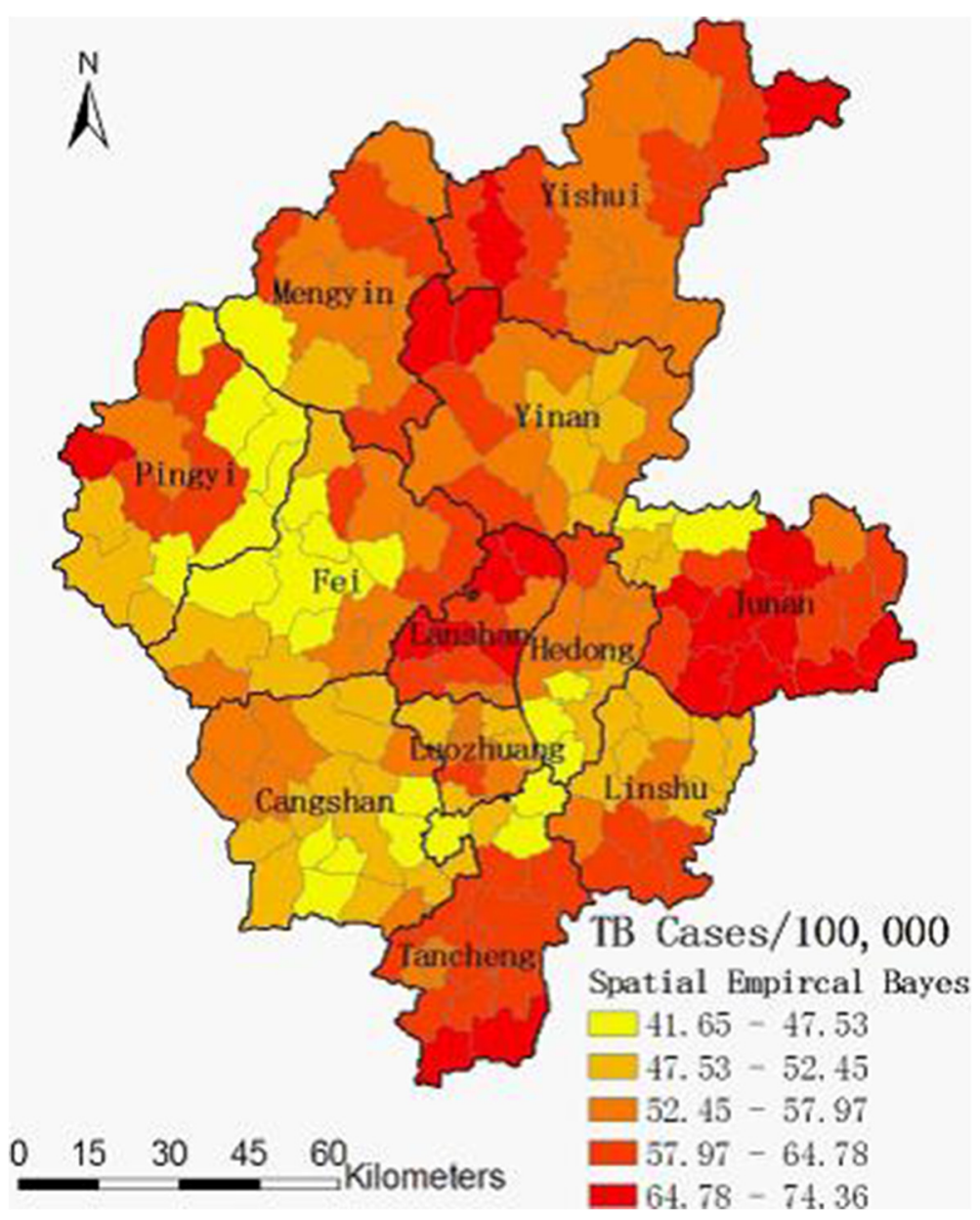

Figure 4 Spatially smoothed map of TB in Linyi City, China from 2005 to 2010.

clusters of the Local $G_{i}^{*}$ with those of the space-time scan statistic, both methods detected similar and significant high-risk clustering. Consistent results using these two methods, in addition to 6-years of TB case data, and a rate smoothing technique suggest that these results are robust.

The result of the present study provided useful information on the prevailing epidemiological situation of TB in Linyi City. The novel knowledge about the presence

Table 1 Global spatial autocorrelation analyses for annualized incidence of TB in Linyi City from 2005 to 2010

\begin{tabular}{llll}
\hline Year & Moran's $\boldsymbol{I}$ & $\boldsymbol{P}$ value & Pattern \\
\hline 2005 & 0.694550 & 0.001 & Clustered \\
\hline 2006 & 0.626996 & 0.001 & Clustered \\
\hline 2007 & 0.573437 & 0.001 & Clustered \\
\hline 2008 & 0.523944 & 0.001 & Clustered \\
\hline 2009 & 0.449246 & 0.001 & Clustered \\
\hline 2010 & 0.518774 & 0.001 & Clustered \\
\hline
\end{tabular}

Table 2 Spatial clusters (hotspots) of the TB identified by local $G_{i}^{*}$ in Linyi City, China from 2005 to 2010

\begin{tabular}{llll}
\hline Town & $\boldsymbol{G}_{\boldsymbol{i}}^{*}$ & $\boldsymbol{P}$-value & Clusters \\
\hline Pingyi Sub-district & 2.1975 & 0.028 & Hotspot \\
\hline Gaozhuang Town & 2.9938 & 0.001 & Hotspot \\
\hline Xiawei Town & 2.8946 & 0.048 & Hotspot \\
\hline Mamuchi Town & 2.6845 & 0.027 & Hotspot \\
\hline Pingshang Town & 2.8557 & 0.016 & Hotspot \\
\hline Zhuanggang Town & 3.8660 & 0.005 & Hotspot \\
\hline Fangqian Town & 3.3624 & 0.004 & Hotspot \\
\hline Xiangdi Town & 4.5037 & 0.006 & Hotspot \\
\hline Zhubian Town & 3.4762 & 0.005 & Hotspot \\
\hline Shizilu Sub-district & 4.8718 & 0.004 & Hotspot \\
\hline Gaofengtou Town & 2.7618 & 0.027 & Hotspot \\
\hline Guichang Town & 3.0776 & 0.008 & Hotspot \\
\hline Lanshan Sub-district & 2.7226 & 0.03 & Hotspot \\
\hline Baishabu Town & 2.4016 & 0.033 & Hotspot \\
\hline Yitang Town & 3.1462 & 0.024 & Hotspot \\
\hline Zaogoutou Town & 2.9522 & 0.006 & Hotspot \\
\hline
\end{tabular}




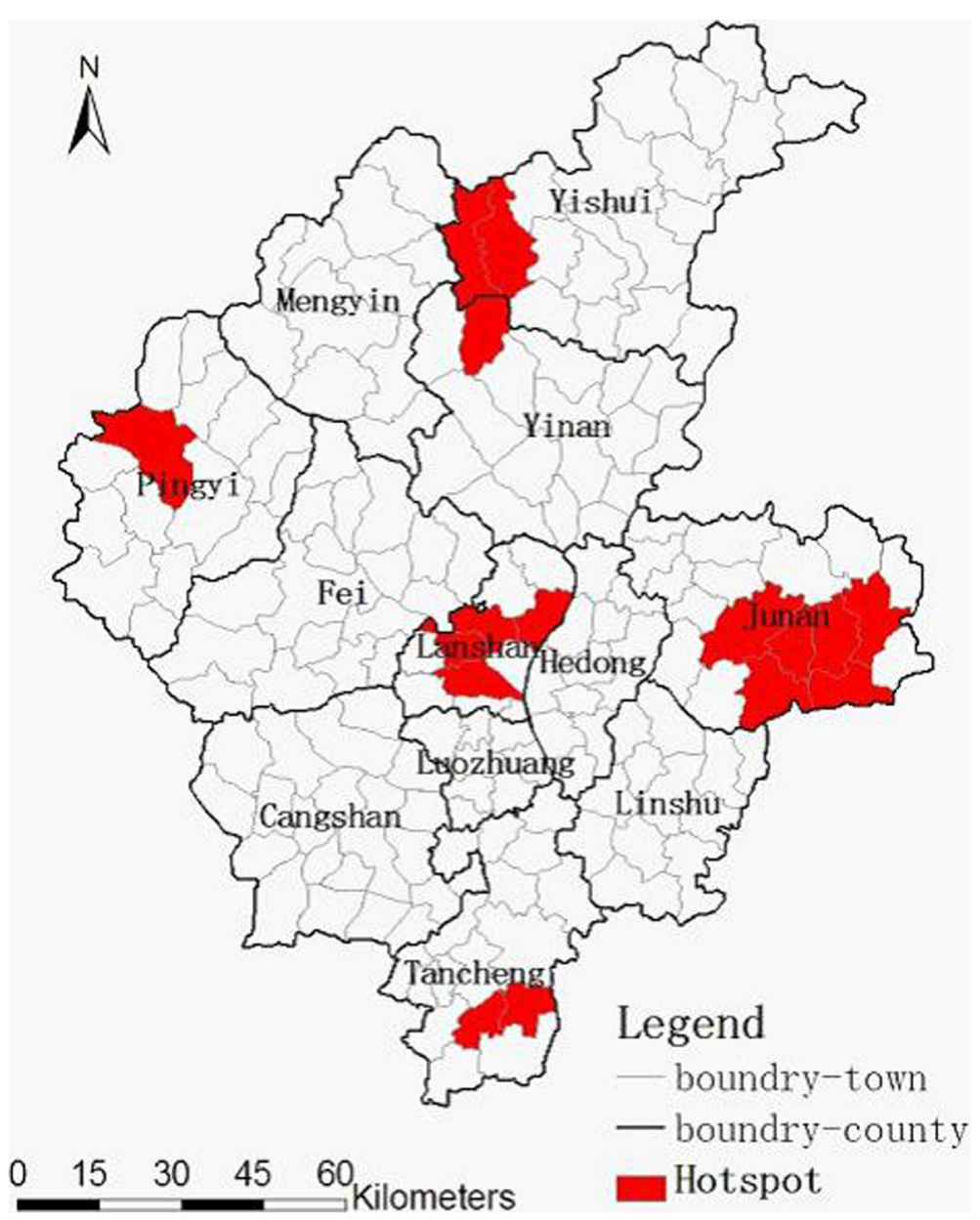

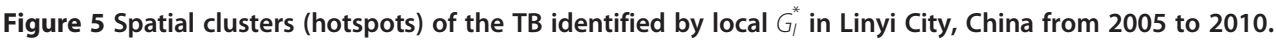

of clusters of TB in Linyi can help the Linyi Institute for Tuberculosis Control to intensify their remedial measures in the identified areas of high tuberculosis prevalence and chalk out future strategies for more effective TB control. Strategies may include compulsory BCG immunization of the children, educating the public about the dangers of TB as a re-emergent epidemic, and monitoring TB carefully.

While our study has demonstrated the usefulness of GIS and spatial analysis, it still has several limitations. First, our data relies on official surveillance and we cannot exclude the possibility that some towns may

Table 3 SaTScan statistics for space-time clusters with significant higher incidence in Linyi City, China from 2005 to 2010

\begin{tabular}{|c|c|c|c|c|c|c|}
\hline Cluster type & Time frame & Cluster areas $(n)$ & Observed cases & Expected cases & Relative risk & Pc $\mathrm{p}$ value \\
\hline Most likely & $2008-2010$ & 1 & 520 & 235.93 & 2.22 & $<0.001$ \\
\hline Secondary & $2007-2009$ & 3 & 715 & 416.87 & 1.73 & $<0.001$ \\
\hline 2nd Secondary & $2008-2010$ & 1 & 358 & 185.34 & 1.94 & $<0.001$ \\
\hline 3rd Secondary & $2008-2010$ & 9 & 793 & 602.50 & 1.32 & $<0.001$ \\
\hline 4th Secondary & $2006-2008$ & 5 & 558 & 409.18 & 1.37 & $<0.001$ \\
\hline 5th Secondary & $2005-2007$ & 1 & 118 & 59.21 & 2.00 & $<0.001$ \\
\hline 6th Secondary & $2007-2009$ & 3 & 408 & 288.18 & 1.42 & $<0.001$ \\
\hline 7th Secondary & $2007-2009$ & 2 & 264 & 173.94 & 1.52 & $<0.001$ \\
\hline 8th Secondary & $2008-2010$ & 5 & 970 & 791.37 & 1.23 & $<0.001$ \\
\hline 9th Secondary & $2008-2010$ & 4 & 490 & 383.61 & 1.28 & 0.003 \\
\hline
\end{tabular}




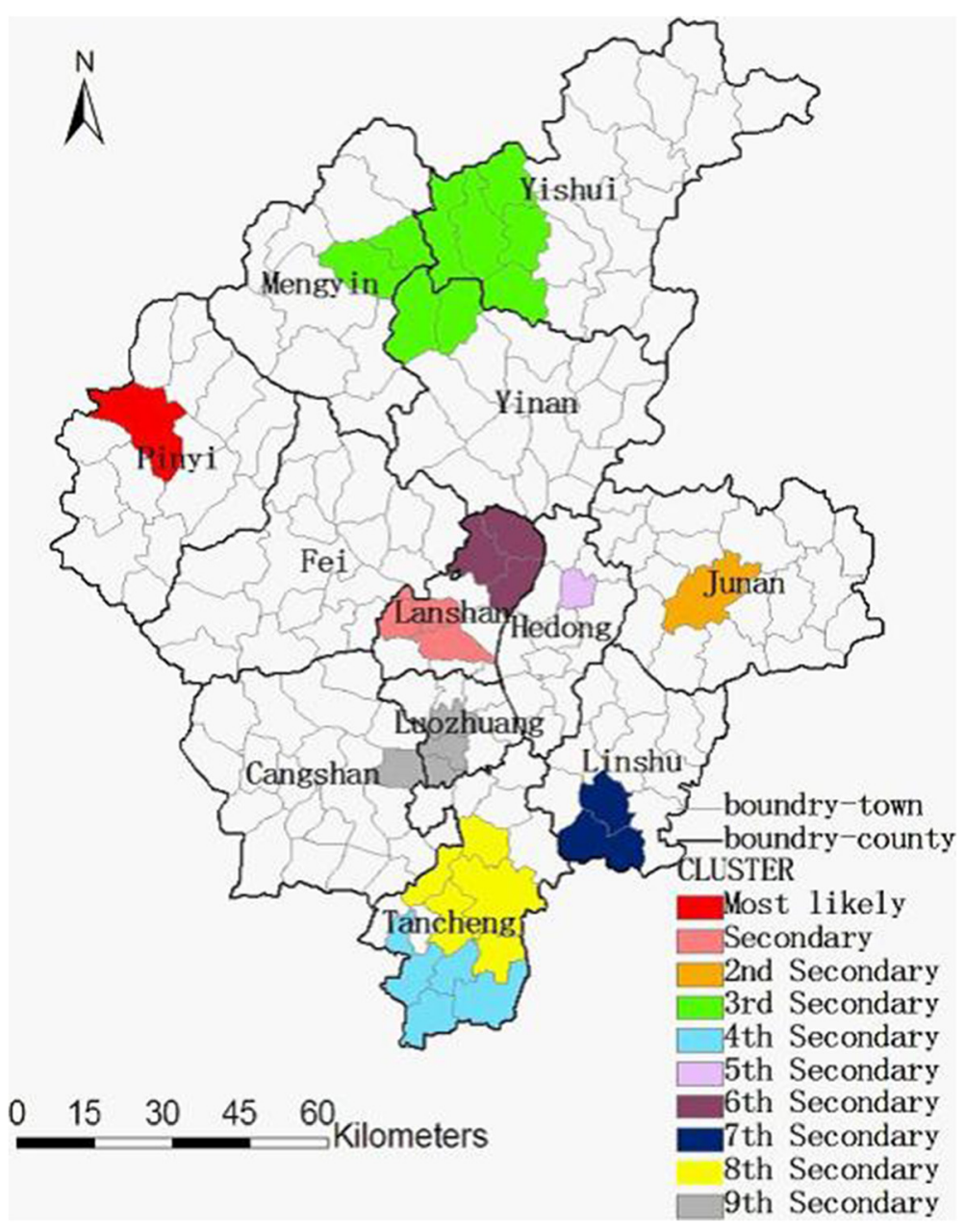

Figure 6 Space-time distribution of the detected clusters of TB cases with significant higher incidences in Linyi City, China from 2005 to 2010 .

underreport the number of cases forvarious reasons. Cases might be missed by routine notification systems because people with TB do not seek care, seek care but remain undiagnosed, or are diagnosed by public and private providers that do not report cases to local or national authorities. Second, we analyzed a relatively short period of time (i.e. 6 years, from 2005 to 2010). Further studies are needed to evaluate the spatial and temporal changes in the pattern of TB using data from a longer study period. Third, we did not assess possible risk factors that could be associated with clustering. It is not a survey-based study but official surveillance, as socioeconomic and environmental factors are not taken into account.

The present study only analyzed the statistically significant clusters of TB. Future researches are warranted to focus on the effect of various socio-economic and environmental factors on the high incidence of TB in the clustering areas. Disease prevalence is frequently associated with many aspects of socio-economic status, such as overcrowding [7], unemployment [7,31], low educational level [32,33], number of shebeens [7] and poor housing quality [34]. Moreover, spatial clustering of TB was also associated with the migrant population [10], patient care factors [5] and environmental factors [7]. After detecting the statistically significant clusters of TB in the region, a survey-based study is intended to identify the role of these factors in the spread of TB.

\section{Conclusion}

This study showed the presence of clusters of TB in Linyi City, China and demonstrated that using the existing health data, the spatial statistic and GIS may provide public health officials with necessary feedback about the prevalence of statistically significant clusters of TB in the region, and thus enable them to carry out more effective strategies to control TB. Since the efficacy of TB control measures in specific areas could be assessed by a longitudinal change in TB prevalence, the space-time 
scan statistic also can contribute to health program evaluation. More detailed individual level investigations are needed in the identified clusters to evaluate the most important determinants of disease distribution.

\section{Competing interests}

The authors declare that they have no competing interests.

\section{Authors' contributions}

TW extracted the data, conducted the statistical analysis and drafted the manuscript. FZX conceived of the project concept, helped to interpret the results and modify the manuscript. YJC helped to interpret the results. YBM extracted the data. YXL conceived of the project concept, assisted with the data interpretation, and helped write the manuscript. All of the authors have read and approved the final manuscript.

\section{Acknowledgements}

This study was supported by the National Natural Science Foundation of China (grant No. 81072340).

\section{Author details}

${ }^{1}$ Department of Epidemiology and Health Statistics, Shandong University, Jinan 250012, People's Republic of China. 'Zibo Municipal Center for Disease Control and Prevention, Zibo 255026, People's Republic of China. ${ }^{3}$ Linyi People's Hospital, Linyi 276000, People's Republic of China.

Received: 21 June 2012 Accepted: 11 October 2012

Published: 19 October 2012

\section{Reference}

1. Maartens G, Wilkinson RJ: Tuberculosis. Lancet 2007, 370(9604):2030-2043.

2. World Health Organization: Global Tuberculosis Control. 2011. http://www who.int/tb/publications/global_report/2011/gtbr11_full.pdf.

3. Tiwari N, Adhikari CM, Tewari A, Kandpal V: Investigation of geo-spatia hotspots for the occurrence of tuberculosis in Almora district, India, using GIS and spatial scan statistic. Int J Health Geogr 2006, 5:33.

4. Onozuka D, Hagihara A: Geographic prediction of tuberculosis clusters in Fukuoka, Japan, using the space-time scan statistic. BMC Infect Dis 2007, 7:26.

5. Randremanana RV, Sabatier P, Rakotomanana F, Randriamanantena A, Richard V: Spatial clustering of pulmonary tuberculosis and impact of the care factors in Antananarivo City. Trop Med Int Health 2009, 14(4):429-437.

6. Nunes C: Tuberculosis incidence in Portugal: spatiotemporal clustering. Int J Health Geogr 2007, 6:30.

7. Munch Z, Van Lill SW, Booysen CN, Zietsman HL, Enarson DA, Beyers N: Tuberculosis transmission patterns in a high-incidence area: a spatial analysis. Int J Tuberc Lung Dis 2003, 7(3):271-277.

8. Touray K, Adetifa IM, Jallow A, Rigby J, Jeffries D, Cheung YB, Donkor S, Adegbola RA, Hill PC: Spatial analysis of tuberculosis in an urban west African setting: is there evidence of clustering? Trop Med Int Health 2010, 15(6):664-672.

9. Maciel EL, Pan W, Dietze R, Peres RL, Vinhas SA, Ribeiro FK, Palaci M, Rodrigues RR, Zandonade E, Golub JE: Spatial patterns of pulmonary tuberculosis incidence and their relationship to socio-economic status in Vitoria, Brazil. Int J Tuberc Lung Dis 2010, 14(11):1395-1402.

10. Jia ZW, Jia XW, Liu YX, Dye C, Chen F, Chen CS, Zhang WY, Li XW, Cao WC, Liu HL: Spatial analysis of tuberculosis cases in migrants and permanent residents, Beijing, 2000-2006. Emerg Infect Dis 2008, 14(9):1413-1419.

11. Clayton D, Kaldor J: Empirical Bayes estimates of age-standardized relative risks for use in disease mapping. Biometrics 1987, 43(3):671-681.

12. Pedigo A, Aldrich T, Odoi A: Neighborhood disparities in stroke and myocardial infarction mortality: a GIS and spatial scan statistics approach. BMC Publ Health 2011, 11:644.

13. Rainey JJ, Omenah D, Sumba PO, Moormann AM, Rochford R, Wilson ML: Spatial clustering of endemic Burkitt's lymphoma in high-risk regions of Kenya. Int J Cancer 2007, 120(1):121-127.

14. Chaikaew N, Tripathi NK, Souris M: Exploring spatial patterns and hotspots of diarrhea in Chiang Mai. Thailand. Int J Health Geogr 2009, 8:36.

15. Tsai PJ, Lin ML, Chu CM, Perng CH: Spatial autocorrelation analysis of health care hotspots in Taiwan in 2006. BMC Publ Health 2009, 9:464
16. Anselin L: Local indicators of spatial association-LISA. Geogr Anal 1995, 27(2):93-115.

17. Moraga P, Montes F: Detection of spatial disease clusters with LISA functions. Stat Med 2011, 30(10):1057-1071.

18. Fang L, Yan L, Liang S, de Vlas SJ, Feng D, Han X, Zhao W, Xu B, Bian L, Yang $H$, et al: Spatial analysis of hemorrhagic fever with renal syndrome in China. BMC Infect Dis 2006, 6:77.

19. Kulldorff M, Feuer EJ, Miller BA, Freedman LS: Breast cancer clusters in the northeast United States: a geographic analysis. Am J Epidemiol 1997, 146(2):161-170.

20. Kulldorff $M$, Nagarwalla N: Spatial disease clusters: detection and inference. Stat Med 1995, 14(8):799-810.

21. Lin H, Liu Q, Guo J, Zhang J, Wang J, Chen H: Analysis of the geographic distribution of HFRS in Liaoning Province between 2000 and 2005. BMC Publ Health 2007, 7:207.

22. Cousens S, Smith PG, Ward H, Everington D, Knight RS, Zeidler M, Stewart G, Smith-Bathgate EA, Macleod MA, Mackenzie J, et al: Geographical distribution of variant Creutzfeldt-Jakob disease in Great Britain, 1994-2000. Lancet 2001, 357(9261):1002-1007.

23. Chen Y, Yi Q, Mao Y: Cluster of liver cancer and immigration: a geographic analysis of incidence data for Ontario 1998-2002. Int J Health Geogr 2008, 7:28.

24. Amin R, Bohnert A, Holmes L, Rajasekaran A, Assanasen C: Epidemiologic mapping of Florida childhood cancer clusters. Pediatr Blood Cancer 2010, 54(4):511-518

25. Wu W, Guo J, Guan P, Sun Y, Zhou B: Clusters of spatial, temporal, and space-time distribution of hemorrhagic fever with renal syndrome in Liaoning Province. Northeastern China. BMC Infect Dis 2011, 11:229.

26. SpaceStat software. http://www.biomedware.com/? module=Page\&s $\mid \mathrm{D}=$ spacestat.

27. Anselin L, Syabri I, Kho Y: GeoDa: An introduction to spatial data analysis. Geogr Anal 2006, 38(1):5-22.

28. SaTScan software. http://www.satscan.org/.

29. Kulldorff M, Athas WF, Feurer EJ, Miller BA, Key CR: Evaluating cluster alarms: a space-time scan statistic and brain cancer in Los Alamos, New Mexico. Am J Public Health 1998, 88(9):1377-1380.

30. Kulldorff M, Heffernan R, Hartman J, Assuncao R, Mostashari F: A space-time permutation scan statistic for disease outbreak detection. PLoS Med 2005, 2(3):e59.

31. Harling G, Ehrlich R, Myer L: The social epidemiology of tuberculosis in South Africa: a multilevel analysis. Soc Sci Med 2008, 66(2):492-505

32. Chan-yeung M, Yeh AG, Tam CM, Kam KM, Leung CC, Yew WW, Lam CW: Socio-demographic and geographic indicators and distribution of tuberculosis in Hong Kong: a spatial analysis. Int J Tuberc Lung Dis 2005, 9(12):1320-1326.

33. Shetty N, Shemko M, Vaz M, D'Souza G: An epidemiological evaluation of risk factors for tuberculosis in South India: a matched case control study. Int J Tuberc Lung Dis 2006, 10(1):80-86.

34. Gustafson P, Gomes VF, Vieira CS, Rabna P, Seng R, Johansson P, Sandstrom A, Norberg R, Lisse I, Samb B, et al: Tuberculosis in Bissau: incidence and risk factors in an urban community in sub-Saharan Africa. Int J Epidemiol 2004, 33(1):163-172.

doi:10.1186/1471-2458-12-885

Cite this article as: Wang et al:: The spatial epidemiology of tuberculosis in Linyi City, China, 2005-2010. BMC Public Health 2012 12:885. 